\title{
An Application of Hankel Transforms in Axially Symmetric Potential Flow
}

\author{
By A. G. Mackie
}

(Received 29th November, 1954.)

In his book on Hydrodynamics, Lamb obtained a solution for the potential flow of an incompressible fluid through a circular hole in a plane wall. More recently Sneddon (Fourier Transforms, New York, 1951) obtained Lamb's solution by an elegant application of Hankel transforms.

Since the streamlines in this solution are symmetric about the wall, it is not of particular physical interest. In this note, Sneddon's method is used to give a solution in which the fluid is infinite in extent on one side of the aperture but issues as a jet of finite diameter on the other side.

Cylindrical polar coordinates $r$ and $z$ are chosen with origin at the centre of the aperture such that $z=0$ is the equation of the plane wall and the circular aperture contained in it is defined by $z=0, r<1$. The flow is to be in the direction of increasing $z$.

The velocity potential $\phi$ must satisfy Laplace's equation

$$
\frac{\partial^{2} \phi}{\partial r^{2}}+\frac{1}{r} \frac{\partial \phi}{\partial r}+\frac{\partial^{2} \phi}{\partial z^{2}}=0
$$

If both sides of this equation are multiplied by $r J_{0}(\xi r)$ and integrated with respect to $r$ from 0 to $\infty$ we get

$$
\frac{d^{2} \bar{\phi}}{d z^{2}}-\xi^{2} \bar{\phi}=0
$$

where

$$
\bar{\phi}=\int_{0}^{\infty} r \phi(r, z) J_{0}(\xi r) d r
$$

Hence $\phi=\int_{0}^{\infty} \xi F(\xi) e^{ \pm \xi z} J_{0}(\xi r) d \xi$ is a solution for suitably chosen $F(\xi)$.

The method of setting up the required solution is as follows. We shall find two potential functions $\phi_{1}$ and $\phi_{2}, \phi_{1}$ being the solution when $z \leqslant 0$ and $\phi_{2}$ when $z \geqslant 0$. It is then necessary to ensure that $\phi_{1}=\phi_{2}$ and 
Hankel Transforms in Axially Symmetric Potential Flow 129

$\frac{\partial \phi_{1}}{\partial z}=\frac{\partial \phi_{2}}{\partial z}$ at the aperture $z=0, r<1$. It should perhaps be pointed out that this is in no sense a "patching" process along characteristic surfaces, appropriate only to hyperbolic differential equations, but is merely a device, in which the properties of the elliptic (Laplace) equation are exploited, to ensure that $\phi_{2}$ is the correct continuation of $\phi_{1}$.

$\phi_{1}$ must satisfy the conditions:

(i) $\phi_{1} \rightarrow 0$ as $z^{2}+r^{2} \rightarrow \infty$,

(ii) $\frac{\partial \phi_{1}}{\partial z}=0$ for $z=0$ and $r>1$.

The only independent condition that $\phi_{2}$ must satisfy is that $\frac{\partial \phi_{2}}{\partial z} \rightarrow U$ as $z \rightarrow \infty, U$ being the velocity of the jet at infinity.

Accordingly we try solutions

$$
\begin{aligned}
& \phi_{1}=\int_{0}^{\infty} \xi A(\xi) e^{r z} J_{0}(\xi r) d \xi, \\
& \phi_{2}=K+U z+\int_{0}^{\infty} \xi B(\xi) e^{-\xi z} J_{0}(\xi r) d \xi .
\end{aligned}
$$

The continuity conditions across the aperture and condition (ii) on $\phi_{1}$ then require

$$
\begin{array}{ll}
\int_{0}^{\infty} \xi\{A(\xi)-B(\xi)\} J_{0}(\xi r) d \xi=K & (r<1), \\
\int_{0}^{\infty} \xi^{2}\{A(\xi)+B(\xi)\} J_{0}(\xi r) d \xi=U & (r<1), \\
\int_{0}^{\infty} \xi^{2} A(\xi) J_{0}(\xi r) d \xi=0 & (r>1) .
\end{array}
$$

These conditions are satisfied by choosing

and

$$
A(\xi)=\frac{K}{\pi} \frac{\sin \xi}{\xi^{2}}+\frac{U}{2} \frac{J_{1}(\xi)}{\xi^{2}}
$$

$$
B(\xi)=-\frac{K}{\pi} \frac{\sin \xi}{\xi^{2}}+\frac{U}{2} \frac{J_{1}(\xi)}{\xi^{2}}
$$


This solution gives us a one parameter family of flows for which the infinity velocity of the jet has the value $U$. Analytically it is represented by

$$
\begin{gathered}
\phi_{1}=\frac{K}{\pi} \int_{0}^{\infty} \xi^{-1} \sin \xi e^{\xi z} J_{0}(\xi r) d \xi+\frac{U}{2} \int_{0}^{\infty} \xi^{-1} J_{1}(\xi) e^{\xi z} J_{0}(\xi r) d \xi \\
\phi_{2}=K+U z-\frac{K}{\pi} \int_{0}^{\infty} \xi^{-1} \sin \xi e^{-i z} J_{0}(\xi r) d \xi+\frac{U}{2} \int_{0}^{\infty} \xi^{-1} J_{1}(\xi) e^{-\xi z} J_{0}(\xi r) d \xi
\end{gathered}
$$

The axial velocity, given by $\left[\frac{\partial \phi}{\partial z}\right]_{r=0}$, can be easily obtained.

On evaluating the appropriate integrals we find that

$$
\left[\frac{\partial \phi_{1}}{\partial z}\right]_{r=0}=\left[\frac{\partial \phi_{2}}{\partial z}\right]_{-r=0}=\frac{U}{2}+\frac{U z}{2 \sqrt{z^{2}+1}}+\frac{K}{\pi\left(z^{2}+1\right)} .
$$

This is seen to have the correct form as $z \rightarrow+\infty$ and $-\infty$.

Other properties of the solution are more easily examined by obtaining the stream function $\psi$, related to $\phi$ by the equations

$$
-\frac{1}{r} \frac{\partial \psi}{\partial z}=\frac{\partial \phi}{\partial r}, \quad \frac{1}{r} \frac{\partial \psi}{\partial r}=\frac{\partial \phi}{\partial z}
$$

Simple properties of Bessel functions give the stream function as

$$
\begin{gathered}
\psi_{1}=\frac{K}{\pi} \int_{0}^{\infty} r \xi^{-1} \sin \xi e^{\xi z} J_{1}(\xi r) d \xi+\frac{U}{2} \int_{0}^{\infty} r \xi^{-1} J_{1}(\xi) e^{\xi z} J_{1}(\xi r) d \xi \\
\psi_{2}=\frac{1}{2} U r^{2}+\frac{K}{\pi} \int_{0}^{\infty} r \xi^{-1} \sin \xi e^{-\xi z} J_{1}(\xi r) d \xi-\frac{U}{2} \int_{0}^{\infty} r \xi^{-1} J_{1}(\xi) e^{-\xi z} J_{1}(\xi r) d \xi
\end{gathered}
$$

Now when $z=0$ and $r>1, \psi_{1}=\frac{K}{\pi}+\frac{U}{4}$, a constant, as is required. This follows from the properties of the Weber-Schafheitlin discontinuous integrals. Consequently, if $R$ is the radius of the jet at infinity,

$$
\frac{1}{2} U R^{2}=\frac{K}{\pi}+\frac{U}{4} \quad \text { or } \quad R=\sqrt{\frac{1}{2}+\frac{2 K}{U \pi}} .
$$

The velocity at the edge of the aperture is infinite. This situation is encountered in Lamb's solution and in many similar incompressible flow patterns. Physically the most interesting problem of this type is when the issuing jet has a free surface. This problem has twice been attacked 
Hankel Transforms in Axially Symmetric Potential Flow 131

numerically, first by Trefftz (Zeitschrift für Math. u. Phys., 64, 1916) using an iterative method based on integral equations and then by Southwell and Vaisey [Phil. Trans. Roy. Soc. (A), 240, 1946] using relaxation methods. The above solution is presented here, partly because of its somewhat unorthodox mathematical approach, and partly because it may provide a method of solving the free surface problem by the superposition of perturbation solutions which die out at infinity.

California Institute of Technology, Pasadena, California, U.S.A. 